Draft Version OCtOBER 28, 2018

Preprint typeset using $\mathrm{IAT}_{\mathrm{E}} \mathrm{X}$ style emulateapj v. 5/2/11

\title{
SIMULATIONS OF EARLY BARYONIC STRUCTURE FORMATION WITH STREAM VELOCITY: II. THE GAS FRACTION
}

\author{
Smadar NaOz ${ }^{1,2}$, NaOki Yoshida ${ }^{3}$, Nickolay Y. Gnedin ${ }^{4,5,6}$ \\ Draft version October 28, 2018
}

\begin{abstract}
Understanding the gas content of high redshift halos is crucial for studying the formation of the first generation of galaxies and reionization. Recently, Tseliakhovich \& Hirata showed that the relative "stream" velocity between the dark matter and baryons at the time of recombination - formally a second order effect, but an unusually large one - can influence the later structure formation history of the Universe. We quantify the effect of the stream velocity on the so-called "characteristic mass" - the minimum mass of a dark matter halo capable of retaining most of its baryons throughout its formation epoch - using three different high-resolution sets of cosmological simulations (with separate transfer functions for baryons and dark matter) that vary in box size, particle number, and the value of the relative velocity between the dark matter and baryons. In order to understand this effect theoretically, we generalize the linear theory filtering mass to properly account for the difference between the dark matter and baryonic density fluctuation evolution induced by the stream velocity. We show that the new filtering mass provides an accurate estimate for the characteristic mass, while other theoretical ansatzes for the characteristic mass are substantially less precise.
\end{abstract}

\section{INTRODUCTION}

Gas rich dark matter halos in the early universe serve as a nurturing ground for dwarf galaxies (e.g., Ricotti et al. 2002b,a; Bromm et al. 2002, 1999; Abel et al. 2002; Naoz et al. 2006; Yoshida et al. 2006, 2008; Greif et al. 2010; Clark et al. 2011; Bromm \& Yoshida 2011, and references therein). Their properties are important to quantify, as they are responsible for metal pollution and ionizing radiation at the onset of structure formation (e.g., Shapiro et al. 2004; Ciardi et al. 2006; Hoeft et al. 2006; Gnedin et al. 2008; Okamoto et al. 2008; Trenti \& Stiavelli 2009). More than that, even if the smallest of gas rich halos are too small for efficiently cooling via atomic hydrogen lines and may not host actual galaxies, these "mini-halos" may produce a 21-cm signature in future radio observations (Kuhlen et al. (2006); Shapiro et al. (2006); Naoz \& Barkana (2008) but see Furlanetto \& Oh (2006)) and might block some of the ionizing radiation, causing an overall delay in the initial progress of reionization (e.g., Barkana \& Loeb 2002; Iliev et al. 2003a, 2005; McQuinn et al. 2007). Thus, the evolution of the gas fraction of dark matter halos at various epochs during the early evolution of the universe is of prime importance.

Recently, Tseliakhovich \& Hirata (2010) showed that not only the amplitudes of the dark matter and bary-

\footnotetext{
snaoz@cfa.harvard.edu

1 Institute for Theory and Computation, HarvardSmithsonian Center for Astrophysics, 60 Garden St.; Cambridge, MA, USA 02138

2 CIERA, Northwestern University, Evanston, IL 60208, USA

${ }^{3}$ Department of Physics, University of Tokyo, Tokyo 1130033, Japan

${ }^{4}$ Particle Astrophysics Center, Fermi National Accelerator Laboratory, Batavia, IL 60510, USA

${ }^{5}$ Kavli Institute for Cosmological Physics and Enrico Fermi Institute, The University of Chicago, Chicago, IL 60637 USA

${ }^{6}$ Department of Astronomy \& Astrophysics, The University of Chicago, Chicago, IL 60637 USA
}

onic density fluctuations were different at early times, but also were their velocities. After recombination, the sound speed of the baryons dropped dramatically, while the dark matter velocity remained high - thus, the relative velocity of baryons with respect to the dark matter became supersonic. Tseliakhovich \& Hirata (2010) also showed that this relative velocity between the baryons and the dark matter remained coherent on scales of a few mega-parsec and was of the order of $\sim 30 \mathrm{~km} \mathrm{sec}^{-1}$ at the time of recombination. This relative velocity is often called the "stream velocity" in the literature, and throughout this paper we will use this term. The stream velocity effect has previously been overlooked, because the velocity terms are formally of the second order in the perturbation theory and should be neglected in the linear approximation. However, this second order effect is unusually large, resulting in the numerically nonnegligible suppression of power at mass scales that correspond to the first bound objects in the Universe (e.g., Yoshida et al. 2003a).

Using the Press-Schechter (Press \& Schechter 1974) formalism, Tseliakhovich \& Hirata (2010) showed that the number density of halos is reduced by more than $60 \%$ for halos with $M=10^{6} \mathrm{M}_{\odot}$ at $z=40$. In a subsequent paper, Tseliakhovich et al. (2010) also included the baryonic temperature fluctuations following Naoz \& Barkana (2005). They found that the stream velocity also resulted in much higher "characteristic" mass - the minimum mass for a dark matter halo capable of retaining most of its gas - as compared to the case without the stream velocity (e.g. Naoz \& Barkana 2007). As has been shown in subsequent studies, the stream velocity effect has important implications on the first structures (Stacv et al. 2011; Maio et al. 2011; Greif et al. 2011; Naoz et al. 2012; Fialkov et al. 2011; O'Leary \& McQuinn 2012; Bovy \& Dvorkin 2012) and may also affect the redshifted cosmological 21-cm signal (Dalal et al. 2010; Bittner \& Loeb 2011; Yoo et al. 2011; Visbal et al. 2012; McQuinn \& O'Leary 2012). 
In this paper we explore the effect of stream velocity on the gas fraction in dark matter halos and compare the simulation results to the predictions from the linear theory (e.g. Tseliakhovich et al. 2010). In our first paper (Naoz et al. 2012, hereafter Paper I) we quantified the stream velocity effect on the evolution of the halo mass function with cosmological simulations. We used three different sets of high resolution simulations in order to study the stream velocity effect systematically, thus understanding the overall trends (instead of concentrating on specific halos). We used a set of simulations with different box sizes, particle numbers, and the values for the stream velocity to analyze the suppression of the structure formation as a function of the stream velocity. In Paper I we found that the total number density of halos is suppressed by $\sim 20 \%$ at $z=25$ in regions of the universe that happen to have $v_{\mathrm{bc}}=1 \sigma_{\mathrm{vbc}}$, where $\sigma_{\mathrm{vbc}}$ is the (scale independent) rms fluctuation of the stream velocity on small scales. In rare patches where $v_{\mathrm{bc}}=3.4 \sigma_{\mathrm{vbc}}$, the relative suppression at the same redshift reaches $50 \%$, remaining at or above the $30 \%$ level all the way to $z=11$. Perhaps the most interesting phenomenon that we found was the high abundance of "empty halos", i.e., halos that had their gas fractions below half of the cosmic mean baryonic fraction $\bar{f}_{\mathrm{b}}$. Specifically, we found that for $v_{\mathrm{bc}}=1 \sigma_{\mathrm{vbc}}$ all halos below $10^{5} \mathrm{M}_{\odot}$ are empty at $z \geq 19$. As a result, the high abundance of empty halos can significantly delay the formation of gas rich "minihalos" and the first galaxies. In this paper we investigate the effect of the stream velocity on the gas fraction in halos. In particular, we quantify the dependence of the characteristic mass on the magnitude of the stream velocity.

For completeness we first describe the parameters and initial conditions of our simulations in 92 . We present our results and analysis of the gas fraction in halos and comparison to the linear approximation in Section 3 . Finally we offer a brief discussion in 4

Throughout this paper, we adopt the following cosmological parameters: $\left(\Omega_{\Lambda}, \Omega_{\mathrm{M}}, \Omega_{b}, \mathrm{n}, \sigma_{8}, H_{0}\right)=(0.72,0.28$, $0.046,1,0.82,70 \mathrm{~km} \mathrm{~s}^{-1} \mathrm{Mpc}^{-1}$ ) (Komatsu et al. 2009).

\section{THE SIMULATIONS}

\subsection{Basic Parameters and Settings}

In this work we use a parallel $N$-body/hydrodynamics code GADGET-2 (Springel et al. 2001; Springel 2005). Below we describe the general features of our 3 simulation sets, which are also summarized in table 1.

1. The first set, named " $N=256$ ", uses a total of $2 \times 256^{3}$ dark matter and gas particles within a cubic box of 200 comoving $\mathrm{kpc}$ on a side. To realize statistically significant number of halos in such a small box, we artificially increase gravitational clustering in the simulation by setting $\sigma_{8}=1.4$. We choose this box size so that a $10^{4} \mathrm{M}_{\odot}$ halo is resolved with $\sim 500$ particles - the value needed to estimate the halo gas fraction reliably (Naoz et al. 2009). The gravitational softening is set to be 40 comoving pc, well below the virial radius of a $10^{4} \mathrm{M}_{\odot}$ halo $(\sim 680$ comoving pc). All the simulations in this set are initialized at $z=199$.

2. The second set, named " $N=512$ ", uses a total
TABLE 1

PARAMETERS OF THE SimUlations

\begin{tabular}{lcc}
\hline SIM & $\begin{array}{c}v_{\mathrm{bc}, 0} \\
\mathrm{~km} \mathrm{sec}^{-1}\end{array}$ & $\begin{array}{c}\sigma_{\text {vbc }} \text { stream } \\
\text { velocity }\end{array}$ \\
\hline \hline 256 runs, & $0.2 \mathrm{Mpc}$, & $z_{\text {in }}=199$ \\
\hline \hline $256_{0}$ & 0 & 0 \\
$256_{1 \sigma}$ & 5.8 & 1 \\
$256_{1.7 \sigma}$ & 10 & 1.7 \\
$256_{3.4 \sigma}$ & 20 & 3.4 \\
\hline \hline 512 runs, & $0.7 \mathrm{Mpc}$, & $z_{\text {in }}=199$ \\
\hline \hline $512_{0}$ & 0 & 0 \\
$512_{1 \sigma}$ & 5.8 & 1 \\
$512_{1.7 \sigma}$ & 10 & 1.7 \\
$512_{3.4 \sigma}$ & 20 & 3.4 \\
\hline \hline 768 runs, & $2 \mathrm{Mpc}$, & $z_{\text {in }}=99$ \\
\hline \hline $768_{0}$ & 0 & 0 \\
$768_{1 \sigma}$ & 3 & 1 \\
$768_{1.7 \sigma}$ & 5 & 1.7 \\
$768_{3.4 \sigma}$ & 10 & 3.4 \\
\hline
\end{tabular}

of $2 \times 512^{3}$ dark matter and gas particles within a cubic box with the size of $700 \mathrm{kpc}$. In this set we also artificially increase $\sigma_{8}$ to 1.4 . With these parameters, a halo with 500 dark matter particles has a mass of $\sim 5 \times 10^{4} \mathrm{M}_{\odot}$. The softening length is set to be 68 comoving pc. All the simulations in this set are initialized at $z=199$.

3. The final set of simulations uses $2 \times 768^{3}$ dark matter and gas particles (which we name the " $N=$ $768 "$ set) in a $2 \mathrm{Mpc}$ box, and starts at $z=99$. For these parameters a halo with 500 dark matter particle has a mass of $\sim 10^{5} \mathrm{M}_{\odot}$. The softening length is set to be 0.2 comoving kpc. We use the "correct" value of $\sigma_{8}=0.82$ for this simulation set.

In each simulation set, we explore a range of the values for the stream velocity (see table 1).

\subsection{Initial Conditions}

As has been shown by Naoz et al. (2006) and Naoz \& Barkana (2007), setting up initial conditions for cosmological simulations on small spatial scales is a delicate issue. High accuracy in initial conditions is crucial for accurately predicting the halo mass function in the lowest mass regime $\left(M \lesssim 10^{7} \mathrm{M}_{\odot}\right)$.

Following Naoz et al. (2011), we generate separate transfer functions for dark matter and baryons as described in Naoz \& Barkana (2005). Ideally, the stream velocities should be realized in the initial conditions in a self-consistent way with the transfer functions that are calculated up to the second order in the perturbation theory (c.f. recent studies by O'Leary \& McQuinn 2012; McQuinn \& O'Leary 2012). However, that would require computing transfer functions up to the second order in perturbation theory, and those are not readily available. Instead, similar to all previous simulation studies, we used the transfer functions computed in the linear approximation. We account for that choice in 3.3. when we compare our simulations to the perturbations theory, and thus our results remain self-consistent.

For all runs, glass-like initial conditions were generated using Zel'dovich approximation. For baryons, we 
have used a glass file with positions shifted by a random vector, thus removing artificial coupling between nearby dark matter and gas particles (Yoshida et al. 2003b). We note that we have used the same phases for dark matter and baryons in all of our simulations, since we showed in Paper I that the spatial shift between baryons and dark matter is unimportant.

Tseliakhovich \& Hirata (2010) demonstrated that, while the stream velocity varies in space, its coherence length is quite large, many Mpc. Hence, on scale of our simulation boxes, it can be treated as constant bulk motion of baryons with respect to the dark matter. We include the effect of stream velocity by adding, at the initial redshift, an additional velocity to the $x$ component of the baryons velocity vector. We test a range of values for the stream velocity, which is convenient to quantify in terms of its rms value on small scales, $\sigma_{\mathrm{vbc}}$. Specifically, we test $v_{\mathrm{bc}}=1 \sigma_{\mathrm{vbc}}$ through $v_{\mathrm{bc}}=3.4 \sigma_{\mathrm{vbc}}$ for all the simulations sets (see table 1).

\subsection{Halo Definition}

We locate dark matter halos by running a friends-offriends group finder algorithm with a linking parameter of 0.2 (only for the dark matter component). We use the identified particle groups to find the center of mass of each halo. After the center is located, we calculate density profiles of dark matter and baryons separately, assuming a spherical halo and using 2000 radial bins between $r_{\min }=0 \mathrm{kpc}$ and $r_{\max }=20 \mathrm{kpc}$. Using the density profiles, we find the virial radius $r_{v i r}$ at which the total overdensity is 200 times the mean background density, and compute the mass and the gas fraction of each halo within that radius. Recently, O'Leary \& McQuinn (2012) used an unconventional definition for halos by using the highest baryonic density peaks as the center their halos. This method preferentially results in larger gas fraction for $\sigma_{\mathrm{vbc}} \neq 0$, compare to our more conservative method.

Recently, More et al. (2011) showed that halos identified by the friends-of-friends algorithm enclose an average overdensity that is substantially larger than 200, and its specific value depends on the halo concentration. In our approach we use the friends-of-friends algorithm only to find the center of mass of a halo, and compute the actual halo mass using the spherical overdensity of 200.

We only retain halos that contain at least 500 dark matter particles within their virial radii. The choice allows us to estimate halo masses to about $15 \%$ precision (Trenti et al. 2010) and to estimate halo gas fractions reliably to a similar level of accuracy (Naoz et al. 2009). However, for some of our fit calculations we also include halos with the number of particles as low as 100; if we do that, we assign a lower weight in the fit to these halos according to the resolution study done in $\mathrm{NaOz}$ et al. (2009), see Appendix A for more details.

\section{RESULTS}

Let us consider the various scales involved in the formation of cosmic structure. On large scales gravity dominates other forces and gas pressure can be neglected. On small scales, on the other hand, the pressure dominates gravity and prevents baryon density fluctuations from growing together with the dark matter fluctuations.
The relative force balance at a given time can be characterized by the "filtering scale" (Gnedin \& Hui 1998) - a physical scale above which a small gas perturbation can grow due to gravity overcoming the pressure gradient. In the non-evolving background, the filtering scale coincides with the classical Jeans (1928) scale, but in the expanding universe the two scales typically differ by a significant factor.

Immediately after recombination Compton scattering of Cosmic Microwave Background (CMB) photons on the residual free electrons after cosmic recombination kept the gas temperature coupled to that of the CMB, the Jeans mass was constant in time and equal to the filtering scale. However, at $z \sim 130$, the gas temperature decoupled from the CMB temperature, the Jeans mass began to decrease with time as the gas cooled adiabatically, and the filtering scale lagged behind the Jeans scale.

Based on results from an early numerical simulations, Gnedin (2000) suggested that the filtering mass also describes the largest halo mass whose gas content is significantly suppressed compared to the cosmic baryon fraction. The latter mass scale, commonly called the "characteristic mass", is defined as the halo mass for which the enclosed baryon fraction equals half of the cosmic mean. Thus, the characteristic mass distinguishes between gas-rich and gas-poor halos. Many semianalytical models of dwarfs galaxies use the characteristic mass scale in order to estimate the gas fraction in halos (e.g., Bullock et al. 2000; Benson et al. 2002a, b; Somerville 2002; Bovy \& Dvorkin 2012). Theoretically this sets an approximate minimum value on the mass that can still form stars.

\subsection{Non-linear Behavior: the Characteristic Mass}

For halos, Gnedin (2000) defined a characteristic mass $M_{c}$ for which a halo contains half the mean cosmic baryon fraction $f_{b}$. In his simulation he found the mean gas fraction in halos of a given total mass $M$, and fitted the simulation results to the following formula:

$$
f_{g, \text { calc }}=f_{\mathrm{b}, 0}\left[1+\left(2^{\alpha / 3}-1\right)\left(\frac{M_{c}}{M}\right)^{\alpha}\right]^{-3 / \alpha},
$$

where $f_{\mathrm{b}, 0}$ is the gas fraction in the high-mass limit 7 . In this function, a higher $\alpha$ causes a sharper transition between the high-mass (constant $f_{g}$ ) limit and the lowmass limit (assumed to be $f_{g} \propto M^{3}$ ). Gnedin (2000) found a good fit for $\alpha=1$, with a characteristic mass that in fact equaled the filtering mass by his definition.

The characteristic mass is essentially a non-linear version of the filtering mass, and so it also measures the competition between gravity and pressure. At high masses, where pressure is unimportant, $f_{g} \rightarrow f_{b, 0}$, while the low mass tail is determined by the suppression of gas accretion by gas pressure. Naoz et al. (2009) found that

\footnotetext{
7 Following Naoz et al. (2011) we defined the high-mass bin as the largest $5 \%$ halo mass, or the largest 5 halos (if the larges $5 \%$ consists of less than 5 halos). These halos are usually gas rich and if when we miss them it causes to an underestimate of the gas fraction at the larger mass bin. Naoz et al. (2011) found this method to be consistent with setting a low linking parameter, and with varying the halo radii between $r_{100}, r_{200}$ and $r_{500}$. This way we overcome the disadvantages of assuming a spherical halo, which misses gas rich halos which undergoes mergers.
} 


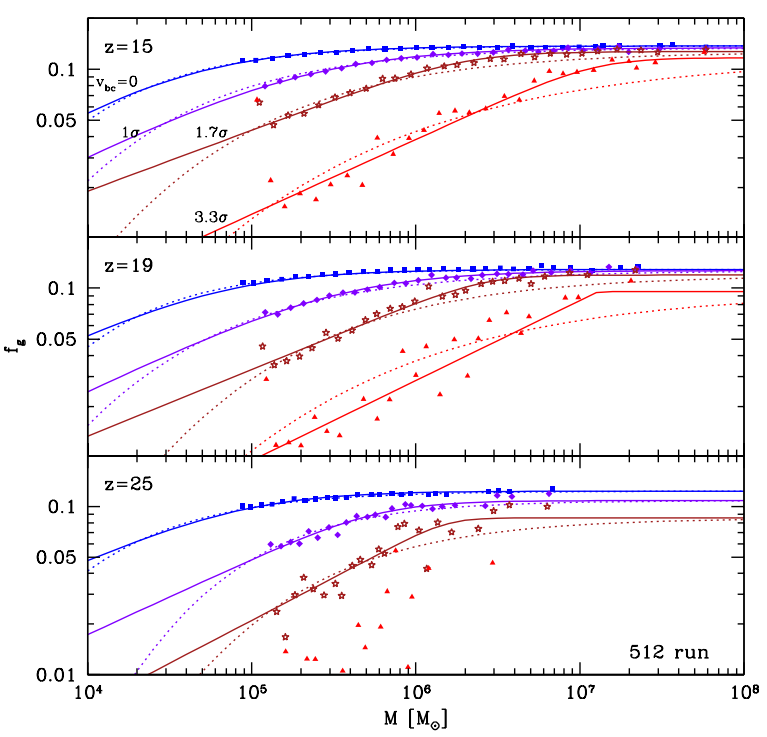

FIG. 1.- Binned gas fraction in the " $\mathrm{N}=512$ " runs at redshifts 25 , 19 , and 15 (from bottom to top panels) for 4 values of the stream velocity $v_{\mathrm{bc}}=0, v_{\mathrm{bc}}=1 \sigma_{\mathrm{vbc}}, v_{\mathrm{bc}}=1.7 \sigma_{\mathrm{vbc}}$, and $v_{\mathrm{bc}}=3.4 \sigma_{\mathrm{vbc}}$ (blue squares, purple diamonds, brown stars and red triangles, respectively). We also show the best fit as evaluated from equations (1) and (2), with dotted and solid lines respectively. Note for the case of $v_{\mathrm{bc}}=3.4 \sigma_{\mathrm{vbc}}$, at $z=25$, about $2 / 3$ of the halos have less than $1 \%$ of gas in them, and $f_{\mathrm{b}, 0}$ is very low. Thus no reliable fit could be found, and therefore we did not show the resulted red line in that case. (See also Figure 7 for the gas fraction behavior for all halos, i.e., not binned.)

the filtering mass from linear theory (calculated in a self consistent way) is consistent with the characteristic mass fitted from the simulations, for two (pre-reionization) scenarios that they tested: a case with no stellar heating and a case of a sudden flash of stellar heating at a given redshift. In a followup paper, Naoz et al. (2011) found the same agreement between the the linear and non-linear theory, and showed that alternative initial conditions models yield a different (higher by about 50 per cent) minimum mass (both the linear and non-linear), since the system retains a memory of the initial conditions. For clarity, we emphasize that the statement $\left(M_{c}=M_{F}\right)$ refers to our definition of $M_{F}$ in equation (9).

In Figure 1 we present the gas fraction as a function of halo mass for our " $\mathrm{N}=512$ " simulation set 8 . As one can see, the halo gas fraction drops dramatically at lower halo masses for large values of the stream velocity. This trend introduces a qualitatively different behavior in the gas fraction as a function of halo mass, which is not captured by equation (11). The best fit ansatz [Eq. (10] is shown with dotted lines in Fig. 1. It clearly does not capture the behavior of the gas fraction as a function of halo mass for $v_{\mathrm{bc}} \neq 0$. Therefore we introduce a new fitting formula for the gas fraction as a function of halo mass,

$$
f_{g, \text { calc }}=f_{\mathrm{b}, 0}\left[1+\left(2^{\gamma}-1\right)\left(\frac{M_{c}}{M}\right)^{\beta}\right]^{-1 / \gamma} .
$$

8 In Paper I we showed that, qualitatively, all of our simulation sets behave similarly as a function of mass and redshift. Therefore, to avoid redundancy, we show here the gas fractions for only "N=512" runs.
The new fitting formula reduces to Equation (1) for $\gamma=$ $3 \beta=\alpha$.

Although we add another free parameter to the fit, finding the best fit model presents some hurdles which we discuss in the Appendix. The best-fit value of $M_{c}$ from Equation (2) is the same as the value from Equation (11) for the $v_{\mathrm{bc}}=0$ case, as can be expected from the fact that Equation (11) provides a good fit to the simulation results in the $v_{\mathrm{bc}}=0$ case. It is interesting, however, that the best fit values for $M_{c}$ from Equation (2) are consistent with the best fit values of $M_{c}$ from Equation (11), even for large values of $v_{\mathrm{bc}}$, as can be seen in Figure 8. However, the new fitting formula gives a better overall fit for high $v_{\mathrm{bc}}$ values, especially in the high mass limit.

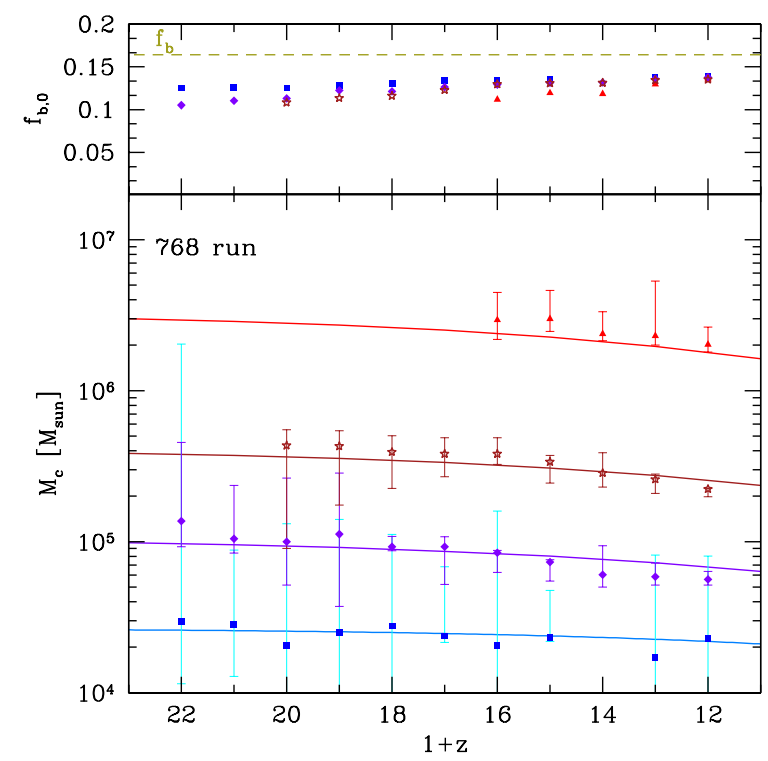

FIG. 2.- Best fit values for the characteristic mass and limiting baryon fraction $f_{\mathrm{b}, 0}$ as functions of redshift for various values of the stream velocity $v_{\mathrm{bc}}=0, v_{\mathrm{bc}}=1 \sigma_{\mathrm{vbc}}, v_{\mathrm{bc}}=1.7 \sigma_{\mathrm{vbc}}$, and $v_{\mathrm{bc}}=3.4 \sigma_{\mathrm{vbc}}$ (blue squares, purple diamonds, brown stars and red triangles respectively) for our $N=762$ simulation set. We also show the evolution of the fully self-consistent filtering mass (Equation 7) with solid curves. The error bars are the maximum $1-\sigma$ from the two fit models (see Appendix A).

Figures 24 show the best-fit values for the characteristic mass and limiting baryon fraction $f_{\mathrm{b}, 0}$ at a range of redshifts. At the highest redshifts and large values of $\sigma_{\mathrm{vbc}}$ most of the halos are empty halos (i.e., halos with gas fraction lower then the half of the mean cosmic baryonic fraction, see Paper I, figures 2-4). This of course means that there is no apparent trend similar to Figure 11. and therefore, no convergence could be achieved in the fitting procedure and the parameters of the fit cannot be measured. Furthermore, as has been noted in Paper I, the $N=256$ set suffers from poor statistics for $M \gtrsim 10^{5} \mathrm{M}_{\odot}$, resulting in large error bars for the best-fit values of the parameters. We show the results of this run to caution the interpretations of previous (and perhaps some future) which employ very small boxes.

An important point to make is that at low redshifts the baryon fraction at the highest mass bins (for all runs) approaches the same value irrespectively of the magnitude of the stream velocity. This is, of course, expected, as the 


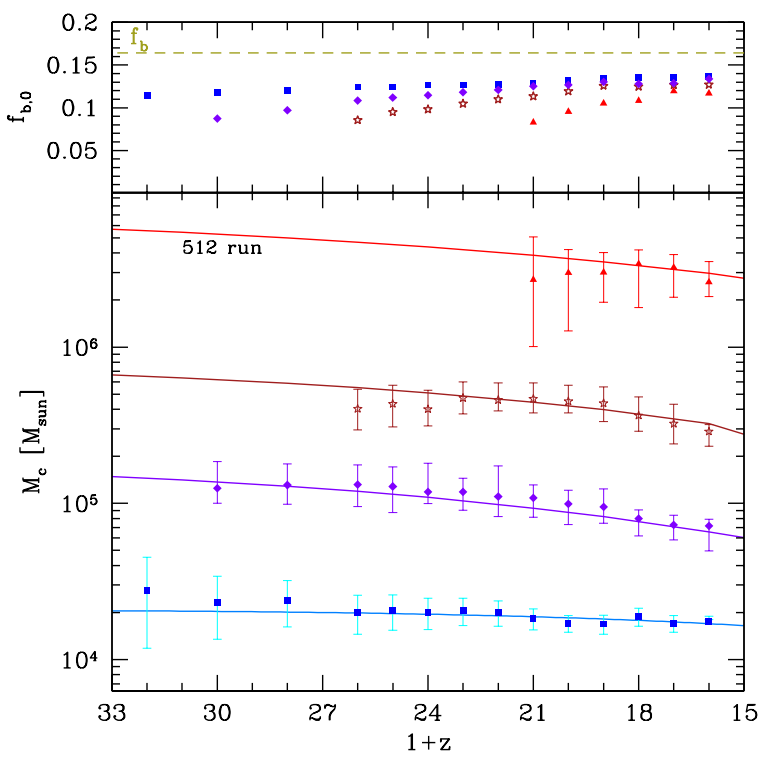

Fig. 3.- Same as Figure 2 but for the $N=512$ simulation set.

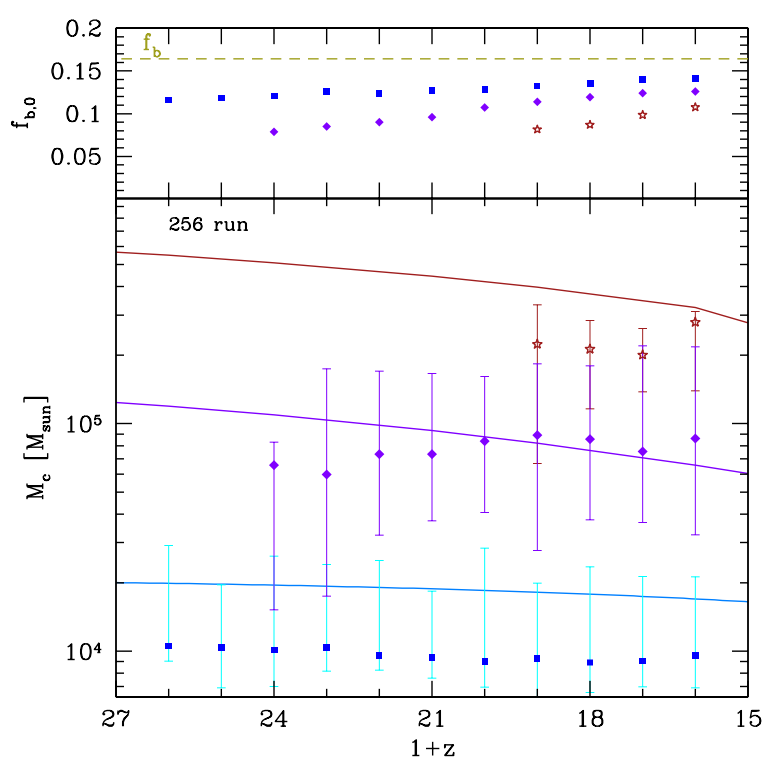

Fig. 4.- Same as Figure 2 but for the $N=256$ simulation set.

global trend for the baryon fraction is to approach the cosmic mean (see Appendix A, Figure 9), but still below it at high redshift (even at large scales). This is because the baryons still did not fall into the Dark Matter potential wells (see Naoz \& Barkana 2007; Barkana \& Loeb 2011, and the Appendix for further discussion). However, in the case of the largest stream velocity we consider, $v_{\mathrm{bc}}=3.4 \sigma_{\mathrm{vbc}}$, the $N=256$ and $N=512$ simulation sets do not quite reach the cosmic mean values expected. This is most likely due to the low abundance of the most massive halos, since some baryons are in fact bound to the halo and we miss them due to our halo finder algorithm (2.3), as can be seen in Figure 4 of Paper I. The $N=768$ simulation set, however, does not suffer from that incomplete convergence. We find a simple fit for the gas fraction in the last mass bin, $f_{\mathrm{b}, 0}$, for the low redshift limit as a function of the stream velocity

$$
f_{\mathrm{b}, 0}=-0.0049 \frac{v_{\mathrm{bc}}}{\sigma_{\mathrm{vbc}}}+0.1345 .
$$

Note that throughout the paper we compare between different simulations using different $\sigma_{8}$ values (i.e., $\sigma_{8}=$ 0.82 for the $N=768$ set and $\sigma_{8}=1.4$ for the $N=512$ and $N=256$ sets). As was shown in Paper I the suppression of the halo mass function, due to the stream velocity, compare to the no stream velocity case is independent on $\sigma_{8}$ (see Figures 5 and 6 in Paper I). Since the characteristic mass describes a relative suppression of the gas fraction in small scales compare to large scales, we find that this quantity is independent on $\sigma_{8}$ as well (where the different simulations gave a consistent value of $M_{c}$ ). This is further supported by the agreement to linear theory (see below). Thus, increasing $\sigma_{8}$ only raised the clustering amplitude that enlarges the sample of simulated halos.

\subsection{Linear Theory Predictions: the Filtering Mass}

In the linear approximation, the filtering mass, first defined by Gnedin \& Hui (1998), describes the highest mass scale on which the baryon density fluctuations are suppressed significantly compared to the dark matter fluctuations. Gnedin \& Hui (1998) only considered the low redshift case, where the baryonic and dark matter fluctuations have the same amplitude at large scales. Naoz \& Barkana (2007) relaxed that assumption and extended the computation of the filtering mass to early times, during which the amplitude of the baryonic fluctuations is below the amplitude of the dark matter fluctuations even on large scales. Both studies, however, only considered a case of zero stream velocity.

In order to extend the derivation of the filtering mass to the non-zero stream velocity case, we first introduce the coupled second order differential equations that govern the evolution of the density fluctuations of the dark matter $\left(\delta_{\mathrm{dm}}\right)$, and the baryons $\left(\delta_{\mathrm{b}}\right)$ and the baryon temperature $\left(\delta_{T}\right)$ :

$$
\begin{aligned}
\ddot{\delta}_{\mathrm{dm}}+ & 2 H \dot{\delta}_{\mathrm{dm}}-f_{\mathrm{dm}} \frac{2 i}{a} \mathbf{v}_{\mathrm{bc}} \cdot \mathbf{k} \dot{\delta}_{\mathrm{dm}}= \\
& \frac{3}{2} H_{0}^{2} \frac{\Omega_{m}}{a^{3}}\left(f_{\mathrm{b}} \delta_{\mathrm{b}}+f_{\mathrm{dm}} \delta_{\mathrm{dm}}\right)+\left(\frac{\mathbf{v}_{\mathrm{bc}} \cdot \mathbf{k}}{a}\right)^{2} \delta_{\mathrm{dm}} \\
\ddot{\delta}_{\mathrm{b}}+2 H \dot{\delta}_{\mathrm{b}} & = \\
\frac{3}{2} H_{0}^{2} \frac{\Omega_{m}}{a^{3}}\left(f_{\mathrm{b}} \delta_{\mathrm{b}}+f_{\mathrm{dm}} \delta_{\mathrm{dm}}\right) & -\frac{k^{2}}{a^{2}} \frac{k_{B} \bar{T}}{\mu}\left(\delta_{\mathrm{b}}+\delta_{T}\right)
\end{aligned}
$$

where $\Omega_{m}$ is the present day matter density as a fraction of the critical density, $k$ is the comoving wavenumber, $a$ is the scale factor, $\mu$ is the mean molecular weight, $H_{0}$ is the present day value of the Hubble parameter $H$, and $\bar{T}$ and $\delta_{T}$ are the mean baryon temperature and its dimensionless fluctuation, respectively. These equations are a compact form of equations 5 in Tseliakhovich \& Hirata $(2010)$, where we used the fact that $v_{\mathrm{bc}} \propto 1 / a$, and the baryon equation includes the pressure term whose form comes from the equation of state of an ideal gas. The linear evolution of the temperature fluctuations is given 
by (Barkana \& Loeb 2005; Naoz \& Barkana 2005)

$\frac{d \delta_{T}}{d t}=\frac{2}{3} \frac{d \delta_{\mathrm{b}}}{d t}+\frac{x_{e}(t)}{t_{\gamma}} a^{-4}\left\{\delta_{\gamma}\left(\frac{\bar{T}_{\gamma}}{\bar{T}}-1\right)+\frac{\bar{T}_{\gamma}}{\bar{T}}\left(\delta_{T_{\gamma}}-\delta_{T}\right)\right\}$,

where $x_{e}(t)$ is the free electron fraction as a function of cosmic time $t, \delta_{\gamma}$ is the photon density fluctuation, $t_{\gamma}=8.55 \times 10^{-13} \mathrm{yr}^{-1}$, and $T_{\gamma}$ and $\delta_{T_{\gamma}}$ are the mean photon temperature and its dimensionless fluctuation, respectively. Equation (6) describes the evolution of the gas temperature in the post-recombination era, but before formation of first galaxies, when the only external heating arises from Compton scattering of the remaining free electrons on the CMB photons. The first term in Equation (6) comes from the adiabatic cooling or heating of the gas, while the second term is the result of the Compton interaction.

In the top right panel of Figure 5 we show an example of the solution of Equations (4). We plot the ratio for $\delta_{\mathrm{b}} / \delta_{\text {tot }}$ as a function of the wavenumber $k$ for the fully self-consistent linear calculation, i.e., starting at the time of recombination and using the exact transfer functions from Tseliakhovich \& Hirata (2010). We consider cases with $v_{\mathrm{bc}}=0, v_{\mathrm{bc}}=\sigma_{\mathrm{vbc}}$, and $v_{\mathrm{bc}}=2 \sigma_{\mathrm{vbc}}$ at $z=10$ and $z=25$. For larger $v_{\mathrm{bc}}$ values, the drop in $\delta_{\mathrm{b}} / \delta_{\text {tot }}$ occurs at larger scales, i.e., the suppression of the baryonic perturbations relative to the total matter fluctuations shifts to larger masses.

Naoz et al. (2009) and Naoz et al. (2011), showed that the characteristic mass is in a good agreement with the filtering mass, regardless of the initial conditions, or even if heating is involved, as long as the filtering mass is calculated self consistently. Motivated by these results we set to find a filtering mass that can be calculated self consistently and that will present the excepted agreement with the simulations. Following Naoz \& Barkana (2007), we re-define the filtering scale (specifically, the filtering wavenumber $k_{F}$ ) to include the stream velocity effect as

$$
\frac{\delta_{\mathrm{b}}}{\delta_{\mathrm{tot}}}=1+r_{\mathrm{LSS}}-\frac{k^{2}}{k_{F}^{2}} \frac{1}{1+\nu},
$$

where $\nu=v_{\mathrm{bc}} / \sigma_{\mathrm{vbc}}$ and $\sigma_{\mathrm{vbc}}$ is the (scale-independent) rms of the stream velocity at small scales. The parameter $r_{\text {LSS }}$ (a negative quantity) describes the relative difference between $\delta_{\mathrm{b}}$ and $\delta_{\text {tot }}$ on large scales (Naoz \& Barkana 2007), i.e.,

$$
r_{\mathrm{LSS}} \equiv \frac{\Delta}{\delta_{\mathrm{tot}}}
$$

where $\Delta=\delta_{\mathrm{b}}-\delta_{\text {tot }}$ (see also Barkana \& Loeb 2005). The filtering mass is defined from $k_{F}$ simply as:

$$
M_{F}=\frac{4 \pi}{3} \overline{\rho_{0}}\left(\frac{1}{2} \frac{2 \pi}{k_{F}}\right)^{3},
$$

where $\overline{\rho_{0}}$ is the mean matter density today.

To find $k_{F}$ in a general case, we write it in the form

$$
k_{F}^{2}(t)=\frac{\delta_{\text {tot }}}{u(t)},
$$

where $u(t)$ is to be determined. Then, using equation (7), we expand the baryonic fluctuation as a function of wavenumber $k$,

$$
\delta_{\mathrm{b}}=\delta_{\mathrm{tot}}+\Delta_{\mathrm{LSS}}-\frac{u(t) k^{2}}{1+\nu}+O\left(k^{4}\right),
$$

where $\Delta_{\text {LSS }} \equiv r_{\text {LSS }} \delta_{\text {tot }}[$ eq. (8) $]$ obeys the following equation to the first order of $k$,

$$
\ddot{\Delta}_{\mathrm{LSS}}+2 H \dot{\Delta}_{\mathrm{LSS}}=-\frac{2 i}{a} f_{\mathrm{dm}} \mathbf{v}_{\mathrm{bc}} \cdot \mathbf{k} \dot{\delta}_{\mathrm{dm}} .
$$

Note that in the case of $v_{\mathrm{bc}}=0$, the linear term of $k$ has a zero coefficient, and thus the right hand side of this equation is simply zero (see Barkana \& Loeb 2005; Naoz \& Barkana 2007). Substituting the expansion from equation (11) into equation (6), and using equations (4) and (12), we obtain an equation for $u$ :

$$
\begin{aligned}
\ddot{u}+2 H \dot{u} & =f_{\mathrm{dm}}(1+\nu)\left\{\frac{1}{a^{2}} \frac{k_{B} \bar{T}}{\mu}\left(\delta_{\mathrm{b}}+\delta_{T}\right)\right. \\
& \left.+\left(\frac{\mathbf{v}_{\mathrm{bc}} \cdot \mathbf{k}}{a}\right)^{2} \delta_{\mathrm{dm}}\right\} .
\end{aligned}
$$

In the limit of $v_{\mathrm{bc}}=0$ (i.e., $\nu=0$ ) this equation reduces to equation (12) of Naoz \& Barkana (2007), and thus results in the same filtering mass found in that study. We can solve Equation (13) to find $u(t)$,

$$
\begin{aligned}
& u(t)= \\
& f_{\mathrm{dm}}(1+\nu)\left\{\int_{t_{\mathrm{rec}}}^{t} \frac{d t^{\prime \prime}}{a^{2}\left(t^{\prime \prime}\right)} \int_{t_{\mathrm{rec}}}^{t^{\prime \prime}} d t^{\prime} \frac{k_{B} \bar{T}\left(t^{\prime}\right)}{\mu}\left(\delta_{\mathrm{b}}\left(t^{\prime}\right)+\delta_{T}\left(t^{\prime}\right)\right)\right. \\
& \left.+\int_{t_{\mathrm{rec}}}^{t} \frac{d t^{\prime \prime}}{a^{4}\left(t^{\prime \prime}\right)} \int_{t_{\mathrm{rec}}}^{t^{\prime \prime}} d t^{\prime}\left(\mathbf{v}_{\mathrm{bc}, \mathrm{rec}}\left(t^{\prime}\right) \cdot \hat{\mathbf{k}}\right)^{2} \delta_{\mathrm{dm}}\left(t^{\prime}\right)\right\} .
\end{aligned}
$$

where $\mathbf{v}_{\mathrm{bc}}$,rec is the stream velocity at the moment of recombination $t_{\mathrm{rec}}$, so that $\mathbf{v}_{\mathrm{bc}}(t)=\mathbf{v}_{\mathrm{bc}, \mathrm{rec}} a\left(t_{\mathrm{rec}}\right) / a(t)$, and $\hat{\mathbf{k}}=\mathbf{k} / k$ is the unit wavenumber vector. In the bottom panel of Figure 5 we show the evolution of the filtering mass as a function of redshift for $v_{\mathrm{bc}}=0,1$ and $2 \sigma_{\mathrm{vbc}}$. The values of the filtering mass for $v_{\mathrm{bc}} \neq 0$ as defined by Equation (77) are larger up to an order of magnitude at high redshifts as compared to the definition of Tseliakhovich et al. (2010). We emphasize that this difference is entirely due to the different definition of the filtering scale, not due to any error in Tseliakhovich et al. (2010) calculations.

The filtering scale $k_{F}$ can also be obtained simply by fitting equation (7) to the calculated values of $\delta_{\mathrm{dm}}$ and $\delta_{b}$, using equations (4)-(6). Naoz \& Barkana (2007) found a functional form that can be used to produce a good fit for the drop of the wavenumber. Generalizing it to the case of stream velocity we write:

$$
\frac{\delta_{\mathrm{b}}}{\delta_{\mathrm{tot}}} \approx\left(1+r_{\mathrm{LSS}}\right)\left(1+\frac{1}{n} \frac{k^{2} / k_{F}^{2}}{1+r_{\mathrm{LSS}}} \frac{1}{1+\nu}\right)^{-n},
$$

and $n$ must be adjusted at each redshift. In the top left panel of Figure 5 we compare this fitting formula to the fully self-consistent linear calculation (long dashed line) for which reproduce the drop of $\delta_{\mathrm{b}} / \delta_{\mathrm{tot}}$ as a function of $k$ fairly well. For the example considered in the figure, i.e., $z=15$ and $v_{\mathrm{bc}}=1 \sigma_{\mathrm{vbc}}$, we find $n=0.46$ and $k_{F}=253.9 \mathrm{Mpc}^{-1}$. We also show the resulted fit using the second order in $k$ approximation, i.e. Eq. (7). 

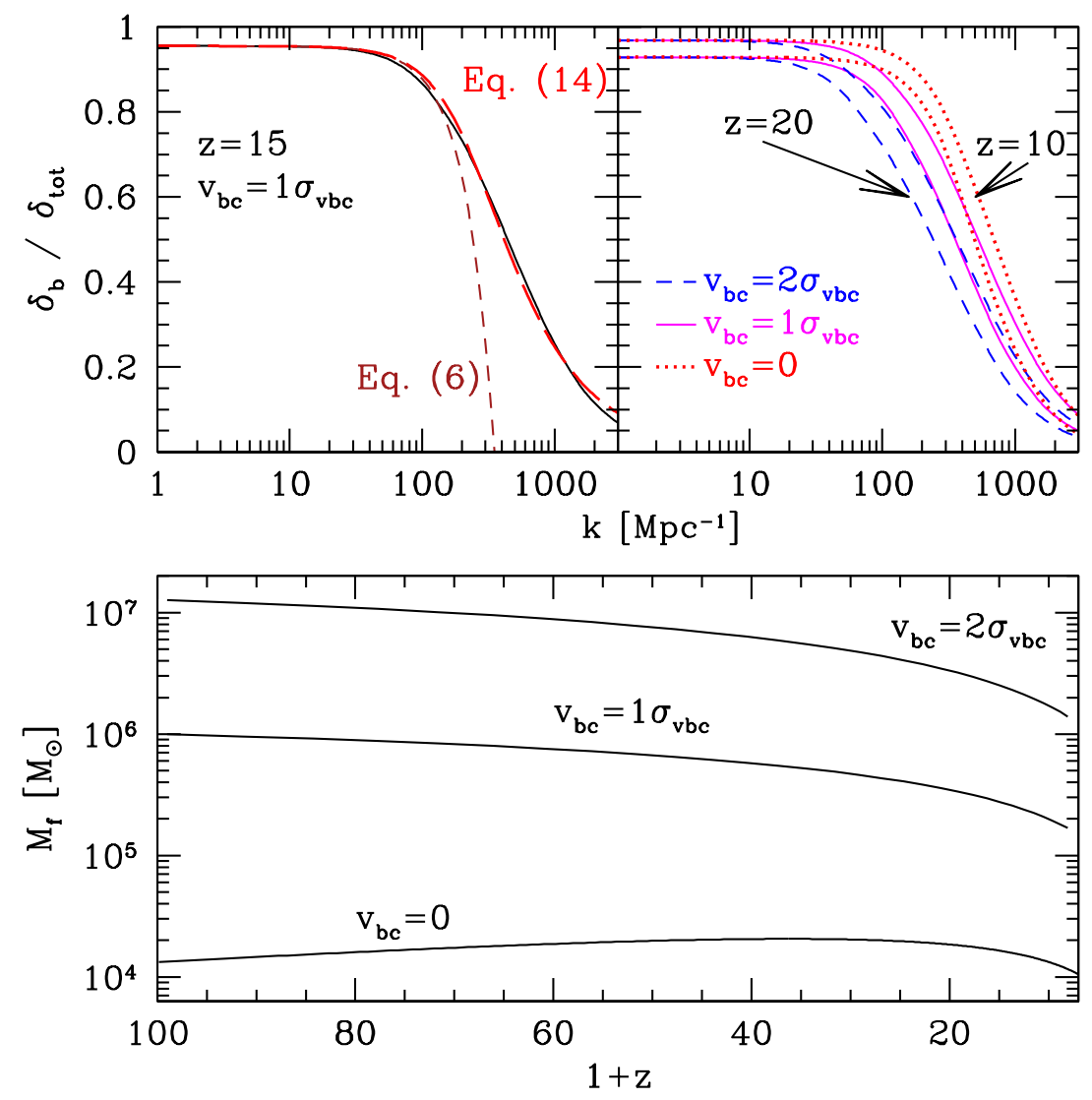

FIG. 5.- Fully self-consistent linear calculation of the growth of the dark matter and baryonic fluctuations (i.e., following Tseliakhovich \& Hirata 2010). We show in the bottom panel the filtering mass as a function of redshift in the regions with $v_{\mathrm{bc}}=0,1$ and $2 \sigma_{\mathrm{vbc}}$ (see labels). In the top panels we show $\delta_{\mathrm{b}} / \delta_{\mathrm{tot}}$ as a function of the wave number $k$. In the right top panel we plot $\delta_{\mathrm{b}} / \delta_{\text {tot }}$ in the regions with $v_{\mathrm{bc}}=0$ (dotted red lines), $1 \sigma_{\mathrm{vbc}}$ (solid magenta lines) and $2 \sigma_{\mathrm{vbc}}$ (dashed blue lines) at $z=10$ (top lines) and $z=25$ (bottom lines), see labels. In the top left panel we show an example for the $v_{\mathrm{bc}}=1 \sigma_{\mathrm{vbc}}$ case at $z=15$ and plot the two fitting functions (see text for details), one which reproduces the drop of $\delta_{\mathrm{b}} / \delta_{\text {tot }}$ with wavenumber, Eq. (15) (red dashed line) and the fitting function to the second order, i.e., Eq. 17), (brown short dashed line).

\subsection{Comparison Between the Linear Theory Predictions and the Nonlinear Results}

In order to compare the filtering mass to the characteristic mass we calculated the filtering mass in a self consistent way, as was done in Naoz et al. (2009, 2011). In other words we use the transfer function from Naoz \& Barkana (2005) with a boosted velocity for the baryons at $z=99(z=199)$ for the $N=768(N=512$ and $N=256)$ set as initial conditions. We then evolve the dark matter and baryon in time according to equations (44)-(6). We note that in all our calculations we included the fact that the boost of the velocity was included in the simulation only in one axis, thus terms which are proportional to $\mathbf{v}_{\mathrm{bc}} \cdot \mathbf{k}$ are reduced by a factor 3 compare to the global average.

Our simulation sets $N=256$ and $N=512$ are initialized at redshift 199 at which Compton heating by the CMB photons significantly affects the evolution of the linear modes and specifically the filtering mass (Naoz \& Barkana 2005, 2007). However, GADGET-2 does not include CMB Compton heating. Hence, to compare apples and apples, we neglected the Compton heating contribution to the filtering mass when comparing these two simulation sets to the linear approxima- tion. This is the reason that our values of the filtering mass in Figures 3 and 4 are lower than the values for the no stream velocity case in Figure 3 of Naoz \& Barkana (2007).

We show the linear theory filtering mass as a function of redshift for all the cases we consider in Figures 24 The filtering mass is consistent with the characteristic mass within our fit errors for all simulation sets. Therefore, we conclude in agreement with $\mathrm{NaOz}$ et al. (2009, 2011), that the evolution of the characteristic mass can be understood using the linear approximation predictions for the filtering mass.

Note that we compare between the filtering mass and $M_{c}$ results from simulation runs using different $\sigma_{8}$. This is possible since the filtering mass definition is independent on $\sigma_{8}$ because it describes the ratio between the two density fluctuations (thus the normalization of the powers simply cancels out).

\subsection{Comparison with the Previous Definition of the Filtering Mass and Other Mass Scales}

Recently Tseliakhovich et al. (2010) showed that including the effects of the relative velocity between the dark matter and the baryons at recombinations results in a higher filtering mass as compared to the case of 


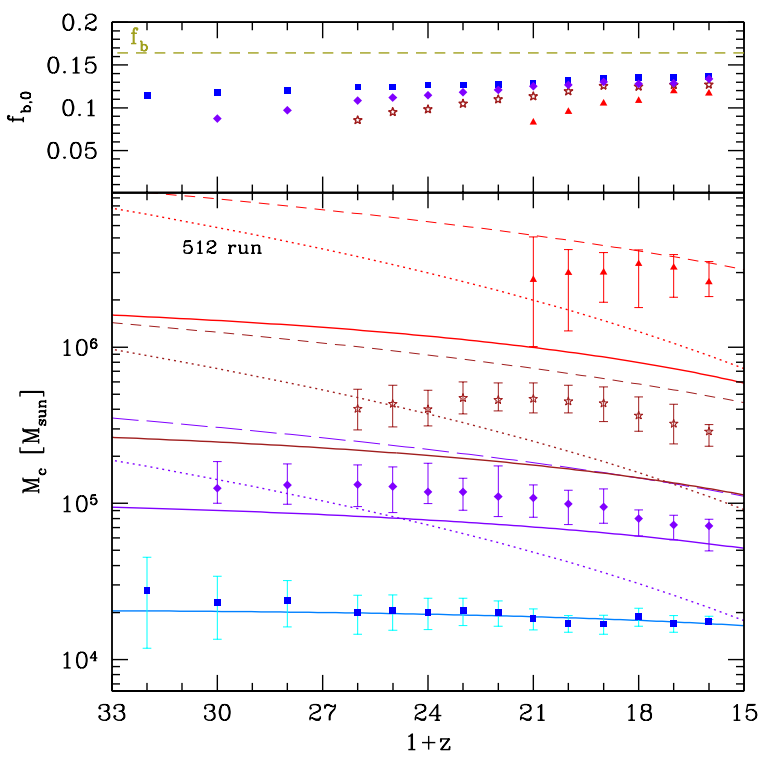

FIG. 6.- Best fit values for the characteristic mass and limiting baryon fraction $f_{\mathrm{b}, 0}$ as functions of redshift (same as in Fig. 3), but now compared with previous theoretical models for the characteristic mass. Solid lines show Naoz \& Barkana (2007) definition of the filtering mass (Eq. 16) that neglects the stream velocity contribution. We also plot $M_{e f f}$ (dashed lines) and $M_{e s c}$ (dotted lines) as alternative models, see text for details.

$v_{\mathrm{bc}}=0$ (by about order of magnitude for the global average). Naoz \& Barkana (2007) defined the filtering scale in the case of $v_{\mathrm{bc}}=0$ as:

$$
\frac{\delta_{\mathrm{b}}}{\delta_{\mathrm{tot}}}=1-\frac{k^{2}}{k_{F}^{2}}+r_{\mathrm{LSS}} .
$$

In Figure 6 6 we show thus defined filtering mass for the $N=512$ simulation set. As can be seen, the Naoz \& Barkana (2007) definition underestimates the characteristic mass in the high $v_{\mathrm{bc}}$ limit, since it neglects the difference between the dark matter and baryons density fluctuations.

Recently Stacy et al. (2011) and Naiman et al. (2011) suggested that, given a high initial stream velocity, the baryon evolution is dominated by the relative motion of dark matter and gas, and thus the gas sound speed should be replaced with the effective sound speed $v_{\text {eff }}$,

$$
v_{\mathrm{eff}}=\sqrt{c_{s}^{2}(z)+v_{\mathrm{bc}}^{2}(z)},
$$

where $v_{\mathrm{bc}}(z)=v_{\mathrm{bc}, 0} /(1+z)$, in the Jeans mass definition. This effective Jeans scale $k_{J \text {,eff }}$ can be written as

$$
k_{J, \mathrm{eff}}=\frac{a}{v_{\mathrm{eff}}} \sqrt{4 \pi G \bar{\rho}_{m}},
$$

where $G$ is the gravitational constant and $\bar{\rho}_{m}$ is the average density. The effective Jean mass $M_{\text {eff }}$ associated with this scale length is simply

$$
M_{\mathrm{eff}}=\frac{4 \pi}{3} \overline{\rho_{0}}\left(\frac{1}{2} \frac{2 \pi}{k_{J, \mathrm{eff}}}\right)^{3} .
$$

We show this mass scale in figures [6 with dashed lines. As one can see, this mass scale overestimates the characteristic mass at all redshifts and for all values of the stream velocity that we considered. This is not unexpected, since the Jeans mass always overestimates the scale at which pressure starts to overtake gravity in the expanding background (Gnedin \& Hui 1998).

The evolution of the characteristic mass as a function of redshift can also be modeled by considering the escape velocity of the gas. Given a mass of a halo $M$, the escape velocity is simply $v_{e s c}=\sqrt{2 G M / r}$, where $r$ is the (comoving) virial radius of the halo. For example, for a halo of $10^{5} \mathrm{M}_{\odot}$ the escape velocity is about $0.77 \mathrm{~km} \mathrm{sec}^{-1}$, while the stream velocity for $v_{\mathrm{bc}}=3.4 \sigma_{\mathrm{vbc}}$ at $z=15$ is $1.6 \mathrm{~km} \mathrm{sec}^{-1}$ and at $z=25$ is $2.6 \mathrm{~km} \mathrm{sec}^{-1}$. Thus, it is not surprising that halos below $10^{5} \mathrm{M}_{\odot}$ are empty in that redshift range - the stream velocity is simply much larger then the halo escape velocity, so the dark matter halo is unable to accrete any gas. We can, thus, estimate a halo mass $M_{\text {esc }}$ below which the stream velocity is larger then the escape velocity,

$$
M_{\mathrm{esc}}=\frac{v_{\mathrm{bc}}(z)^{3}}{\sqrt{\left(2 G H_{0}\right)^{2} \Omega_{m} \Delta_{c}}},
$$

where $\Delta_{c}=200$ is the virial overdensity and $v_{\mathrm{bc}}(z)$ is the stream velocity at redshift $z$. We show this limit in Figure 6 with dotted lines. Equation (20) predicts a much stronger evolution of the characteristic mass than is actually observed in our simulations and provides a poor fit to simulations results.

\section{CONCLUSIONS}

We have used three-dimensional hydrodynamical simulations to investigate the effects of stream velocity on the gas fraction in high redshift halos. In a companion paper Naoz et al. (2012), we studied the effect of the stream velocity on the total halo mass function, In this work we focus on the effect of the stream velocity on the gas fraction in halos and on the evolution of the characteristic mass, and compare the simulation results to the linear approximation.

In a first improvement over the earlier results, we introduce a new fitting formula (Eq. 2) which offers a much better fit to the gas fraction as a function of halo mass at a given redshift in the limit of large stream velocities, while returning essentially the same values of the characteristic mass $M_{c}$ as the previously used functional form (see Figures 1, 7 and 8 , and see Appendix A).

Previous studies (Naoz et al. 2009, 2011) showed that a quantity defined in the linear approximation, the filtering scale (Gnedin \& Huil 1998), provides a good match to the nonlinear characteristic mass measured in numerical simulations. We introduce a new definition for the linear filtering mass that accounts for two effects neglected in Gnedin \& Hui (1998): the deviation of the amplitude of baryonic fluctuations from the dark matter fluctuations on large scales (considered first by Naoz \& Barkana 2007) and the stream velocity between the dark matter and baryons on small scales, which we include in the definition of the filtering mass for the first time in this paper. The latter effect may result in the filtering mass being up to an order of magnitude larger at high redshifts for high values of the stream velocity, as compared to the case when the stream velocity is neglected.

Finally, in comparing our simulations results to the linear calculation (using our new definition of the filtering 
mass), we find that the filtering mass (i.e. a linear quantity) offers an accurate match to the actual nonlinear characteristic mass measured from the simulations, at all redshifts and for all values of the stream velocity that we simulated. On the contrary, previous theoretical models that used as the characteristic mass scale either the halo mass with the escape velocity equal to the stream velocity or the Jeans mass for the "effective" gas sound speed provide only poor fits to the simulation results.

It has been suggested in the literature that gas rich low mass halos may play an important role in cosmic reionization, and that they can produce distinct 21-cm signatures (Kuhlen et al. (2006); Shapiro et al. (2006); Naoz \& Barkana (2008) but see Furlanetto \& Oh $(2006))$. For example, minihalos (halos of mass $\sim$ $\left.10^{6} \mathrm{M}_{\odot}\right)$ can potentially block ionizing radiation and induce an overall delay in the initial progress of reionization (e.g., Shapiro \& Giroux 1987; Barkana \& Loeb 2002; Iliev et al. 2003b; Shapiro et al. 2004; Iliev et al. 2005; McQuinn et al. 2007). However, our results here suggest that at high redshifts the stream velocity effect results in large variations in the characteristic mass - i.e. the minimum mass of a gas rich halo. Thus, if reionization started sufficiently early (Yoshida et al. 2007), in patches of the universe where the stream velocity is large there were fewer gas rich halos that can absorb ionizing photons. Hence, in these patches the delay of the reionization caused by minihalos would be less than in regions that happen to have a small value of the stream velocity and, hence, a large abundance of minihalos. Therefore, not only the formation of the first generations of galaxies may be affected by the stream velocity effect, but also the whole process of reionization may proceed differently in regions with very different stream velocities. This effect has been considered recently by Visbal et al. (2012) and McQuinn \& O'Learv (2012), but our results indicate that it can even be stronger than previously estimated.

\section{ACKNOWLEDGMENTS}

We thank Avi Loeb, Rennan Barkana, Andrey Kravtsov, Neal Dalal, Will Farr, Matt McQuinn and Dmitriy Tseliakhovich for useful discussions. We thank Dmitriy Tseliakhovich for providing his code. We also thank Yoram Lithwick for the use of his allocation time on the computer cluster Quest. This research was supported in part through the computational resources and staff contributions provided by Information Technology at Northwestern University as part of its shared cluster program, Quest.

\section{REFERENCES}

Abel, T., Bryan, G. L., \& Norman, M. L. 2002, Science, 295, 93

Barkana, R. \& Loeb, A. 2002, ApJ, 578, 1

—. 2005, MNRAS, 363, L36

-. 2011, MNRAS, 839

Benson, A. J., Frenk, C. S., Lacey, C. G., Baugh, C. M., \& Cole, S. 2002a, MNRAS, 333, 177

Benson, A. J., Lacey, C. G., Baugh, C. M., Cole, S., \& Frenk, C. S. 2002b, MNRAS, 333, 156

Bittner, J. M. \& Loeb, A. 2011, ArXiv e-prints

Bovy, J. \& Dvorkin, C. 2012, ArXiv e-prints

Bromm, V., Coppi, P. S., \& Larson, R. B. 1999, ApJ, 527, L5

-. 2002, ApJ, 564, 23

Bromm, V. \& Yoshida, N. 2011, ArXiv e-prints

Bullock, J. S., Kravtsov, A. V., \& Weinberg, D. H. 2000, ApJ, 539,517

Ciardi, B., Scannapieco, E., Stoehr, F., Ferrara, A., Iliev, I. T., \& Shapiro, P. R. 2006, MNRAS, 366, 689

Clark, P. C., Glover, S. C. O., Klessen, R. S., \& Bromm, V. 2011, ApJ, 727, 110

Dalal, N., Pen, U., \& Seljak, U. 2010, JCAP, 11, 7

Fialkov, A., Barkana, R., Tseliakhovich, D., \& Hirata, C. M. 2011, ArXiv e-prints

Furlanetto, S. R. \& Oh, S. P. 2006, ApJ, 652, 849

Gnedin, N. Y. 2000, ApJ, 542, 535

Gnedin, N. Y. \& Hui, L. 1998, MNRAS, 296, 44

Gnedin, N. Y., Kravtsov, A. V., \& Chen, H. 2008, ApJ, 672, 765

Greif, T., White, S., Klessen, R., \& Springel, V. 2011, ArXiv e-prints

Greif, T. H., Glover, S. C. O., Bromm, V., \& Klessen, R. S. 2010, ApJ, 716, 510

Hoeft, M., Yepes, G., Gottlöber, S., \& Springel, V. 2006, MNRAS, 371, 401

Iliev, I. T., Scannapieco, E., Martel, H., \& Shapiro, P. R. 2003a, MNRAS, 341, 81

-. 2003b, MNRAS, 341, 81

Iliev, I. T., Scannapieco, E., \& Shapiro, P. R. 2005, ApJ, 624, 491

Jeans, J. H. 1928, Astronomy and cosmogony, ed. Jeans, J. H.

Komatsu, E., Dunkley, J., Nolta, M. R., Bennett, C. L., Gold, B., Hinshaw, G., Jarosik, N., Larson, D., Limon, M., Page, L., Spergel, D. N., Halpern, M., Hill, R. S., Kogut, A., Meyer, S. S., Tucker, G. S., Weiland, J. L., Wollack, E., \& Wright, E. L. 2009, ApJS, 180, 330

Kuhlen, M., Madau, P., \& Montgomery, R. 2006, ApJ, 637, L1
Maio, U., Koopmans, L. V. E., \& Ciardi, B. 2011, MNRAS, L197+

McQuinn, M., Lidz, A., Zahn, O., Dutta, S., Hernquist, L., \& Zaldarriaga, M. 2007, MNRAS, 377, 1043

McQuinn, M. \& O'Leary, R. M. 2012, ArXiv e-prints

More, S., Kravtsov, A. V., Dalal, N., \& Gottlöber, S. 2011, ApJS, 195,4

Naiman, J. P., Ramirez-Ruiz, E., \& Lin, D. N. C. 2011, ApJ, 735, 25

Naoz, S. \& Barkana, R. 2005, MNRAS, 362, 1047

-. 2007, MNRAS, 377, 667

—. 2008, MNRAS, 385, L63

Naoz, S., Barkana, R., \& Mesinger, A. 2009, MNRAS, 399, 369

Naoz, S., Noter, S., \& Barkana, R. 2006, MNRAS, 373, L98

Naoz, S., Yoshida, N., \& Barkana, R. 2011, MNRAS, 416, 232

Naoz, S., Yoshida, N., \& Gnedin, N. Y. 2012, ApJ, 747, 128

Okamoto, T., Gao, L., \& Theuns, T. 2008, MNRAS, 390, 920

O'Leary, R. M. \& McQuinn, M. 2012, ArXiv e-prints

Press, W. H. \& Schechter, P. 1974, ApJ, 187, 425

Ricotti, M., Gnedin, N. Y., \& Shull, J. M. 2002a, ApJ, 575, 33

-. 2002b, ApJ, 575, 49

Shapiro, P. R., Ahn, K., Alvarez, M. A., Iliev, I. T., Martel, H., \& Ryu, D. 2006, ApJ, 646, 681

Shapiro, P. R. \& Giroux, M. L. 1987, ApJ, 321, L107

Shapiro, P. R., Iliev, I. T., \& Raga, A. C. 2004, MNRAS, 348, 753

Somerville, R. S. 2002, ApJ, 572, L23

Springel, V. 2005, MNRAS, 364, 1105

Springel, V., Yoshida, N., \& White, S. D. M. 2001, Nature, 6, 79

Stacy, A., Bromm, V., \& Loeb, A. 2011, ApJ, 730, L1

Trenti, M., Smith, B. D., Hallman, E. J., Skillman, S. W., \& Shull, J. M. 2010, ApJ, 711, 1198

Trenti, M. \& Stiavelli, M. 2009, ApJ, 694, 879

Tseliakhovich, D., Barkana, R., \& Hirata, C. 2010, ArXiv e-prints Tseliakhovich, D. \& Hirata, C. 2010, Phys. Rev. D, 82, 083520

Visbal, E., Barkana, R., Fialkov, A., Tseliakhovich, D., \& Hirata, C. 2012, ArXiv e-prints

Yoo, J., Dalal, N., \& Seljak, U. 2011, JCAP, 7, 18

Yoshida, N., Abel, T., Hernquist, L., \& Sugiyama, N. 2003a, ApJ, 592,645

Yoshida, N., Omukai, K., \& Hernquist, L. 2007, ApJ, 667, L117

-. 2008, Science, 321, 669

Yoshida, N., Omukai, K., Hernquist, L., \& Abel, T. 2006, ApJ, 652,6 


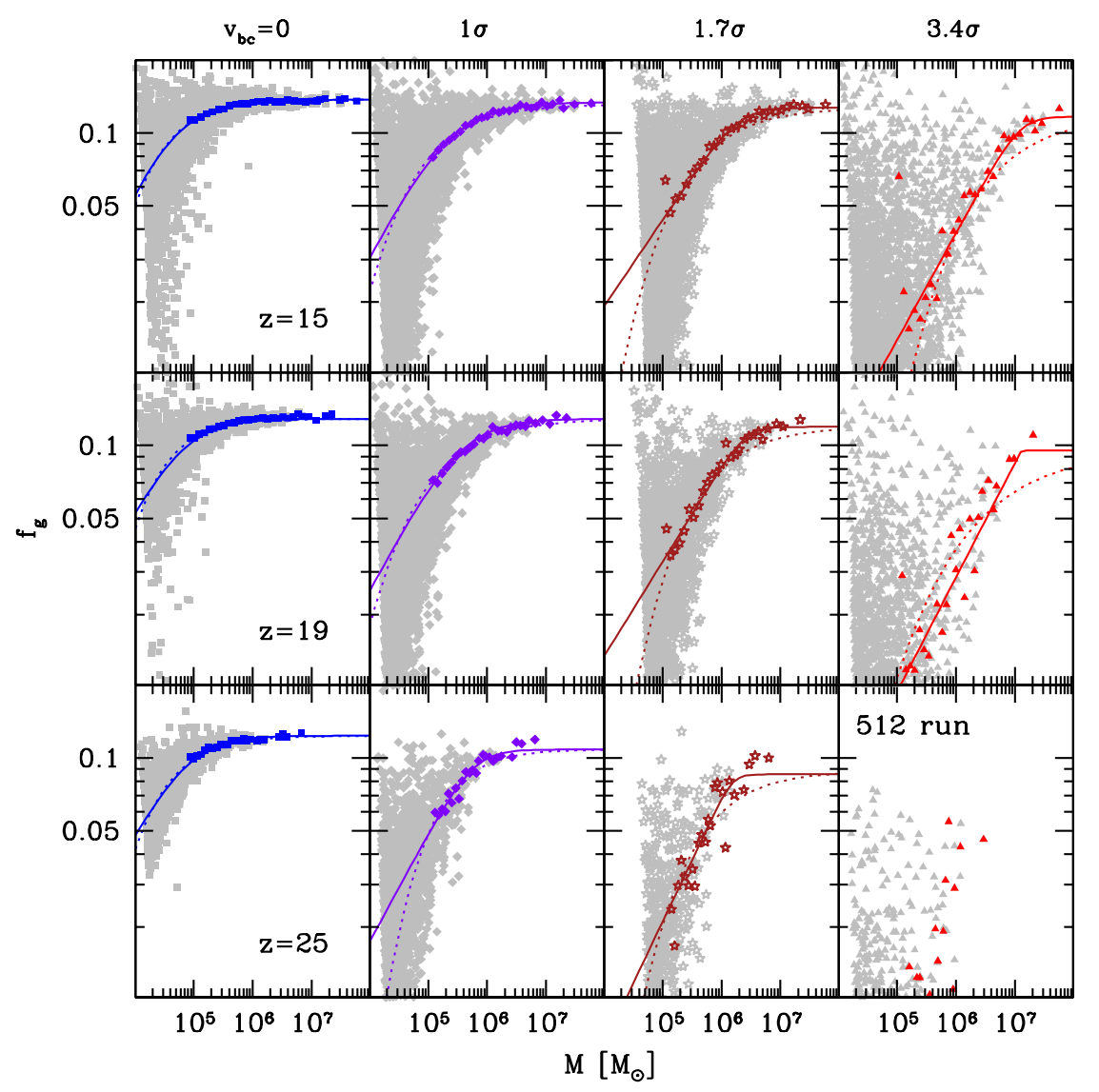

FIG. 7.- A representative example of the gas fraction as a function of mass for the $N=512$ set for three different redshifts (top to bottom) $z=15,19$ and $z=20$. We compare between the new fit model, solid lines, using eq. (2), and the old one, dotted line, using eq. (1 We show all of the halo of which $N_{h} \geq 100$ (grey points) as well as the binned data points for $N_{h} \geq 500$. We consider the various values of the stream velocity (from left to right). $v_{\mathrm{bc}}=0, v_{\mathrm{bc}}=1 \sigma_{\mathrm{vbc}}, v_{\mathrm{bc}}=1.7 \sigma_{\mathrm{vbc}}$, and $v_{\mathrm{bc}}=3.4 \sigma_{\mathrm{vbc}}$. Note for the case of $v_{\mathrm{bc}}=3.4 \sigma_{\mathrm{vbc}}$, at $z=25$, about $2 / 3$ of the halos have less than $1 \%$ of gas in them, and $f_{\mathrm{b}, 0}$ is very low. Thus no reliable fit could be found, and therefore we did not show the resulted red line in that case.

Yoshida, N., Sugiyama, N., \& Hernquist, L. 2003b, MNRAS, 344, 481

\section{APPENDIX}

\section{FIT CALCULATION}

As can see in Figure1 (dotted lines), the fitted formula found by Gnedin (2000), i.e., eq. (1), dose not capture the behavior of the gas fraction as a function of mass for the cases of non-negligible stream velocity. In our search for better fitting formula we found that the low mass tail has a significant effect on the fit for large $v_{\mathrm{bc}}$ values. It is not surprising since the stream velocity deprives the low mass halos of gas at high redshift, as we showed in Paper I. Therefore, this induce a large dependency on the behavior of the gas fraction in the low mass tail. However, the gas fraction of the low mass halos with less than 500 particles is poorly constrained (as was shown in Naoz et al. 2009). Therefore, we introduce a weight function, that account for the errors in estimating the gas fraction as a function of the number of particle in a halo, $N_{h}$. We use Naoz et al. (2009) resolution study, their figure 4, and assume a scatter of $20 \%$ for halos that have more than 500 particles. For smaller number of particles per halo we adopted a simply linear function of the error of the gas fraction as can be estimated from Naoz et al. (2009), figure 4. Thus the weight function has the following form:

$$
\mathcal{W}\left(N_{h}\right)= \begin{cases}0.2 & \text { if } N_{h} \geq 500 \\ 7.5 \times 10^{-4} N_{h}+0.575 & \text { if } N_{h}<500\end{cases}
$$

Using this weight function we find the fit, using the forma formula [eq. (11)] and the new formula eq. (2). In figure [7 we show an example for the gas fraction as a function of mass, and the two fits as in Figure 1 (solid lines for the new formula and dotted lines for the former one). Here we also show all of the points we considered in evaluating the fit (i.e., $\left.N_{h} \geq 100\right)$. We note that the $\chi^{2}$ for the new formula fit is for most cases higher (closer to one) than the $\chi^{2}$ of the old formula, both lower than unity. 


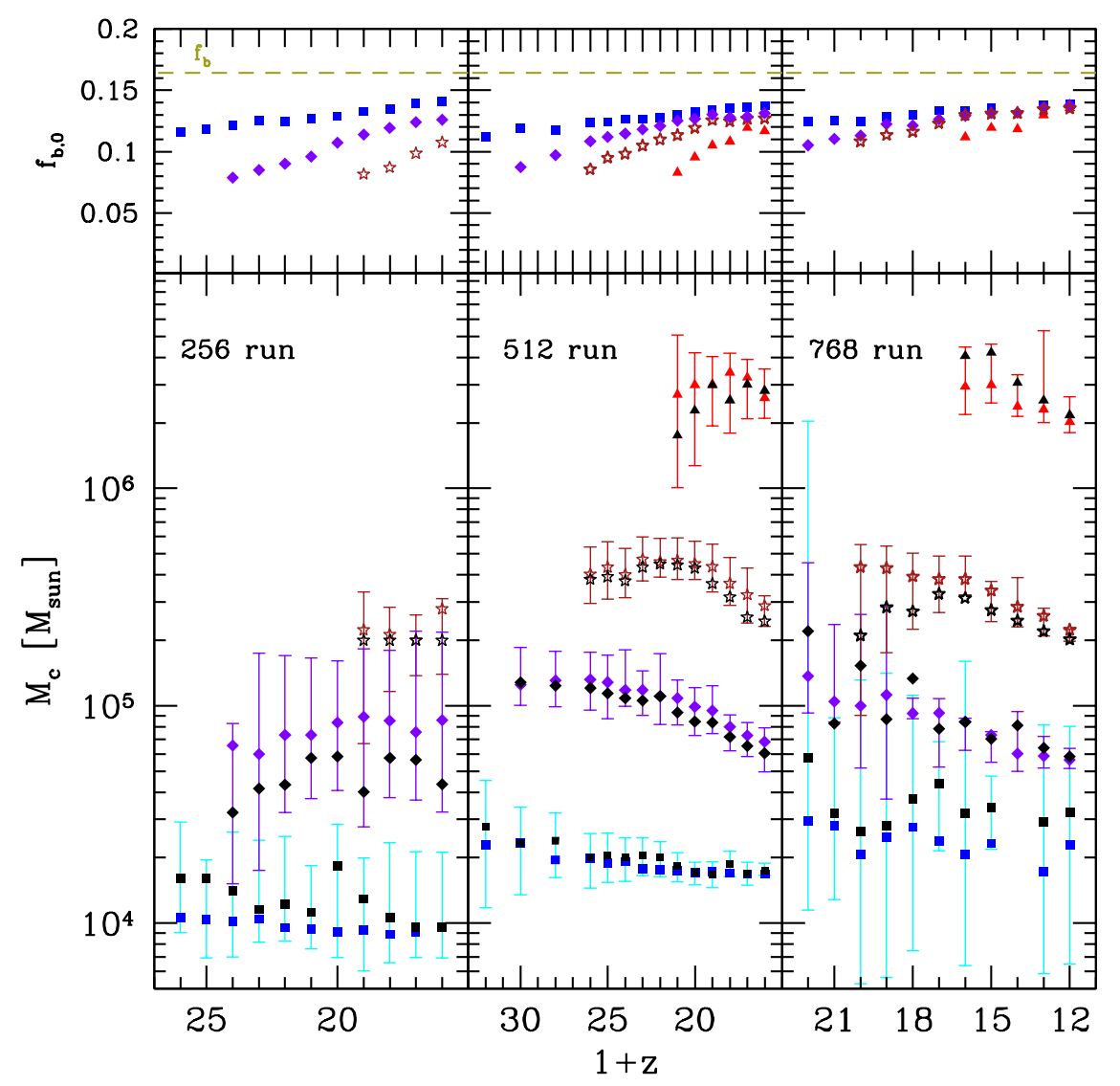

FIG. 8. - Comparison between the new fit model and the old one. We consider in black the old model, using eq. (1), and the color points are the fit for the new model, using eq. (2), calculated with minimum of 100 particles per halo (see text). We consider (from left to right) the $N=256,512$ and $N=768$ sets. We consider the various values of the stream velocity $v_{\mathrm{bc}}=0, v_{\mathrm{bc}}=1 \sigma_{\mathrm{vbc}}, v_{\mathrm{bc}}=1.7 \sigma_{\mathrm{vbc}}$, and $v_{\mathrm{bc}}=3.4 \sigma_{\mathrm{vbc}}$ (blue squares, purple diamonds, brown stars and red triangles respectively).

The ultimate goal of the fitting process is to find the characteristic mass $M_{c}$. An important test is to compare the resulted $M_{c}$ from the two fitting formulae. The different values achieved for the two models are shown in Figure 8 As depicted in this Figure, the $M_{c}$ for the different values of $v_{\mathrm{bc}}$ converge over the different ranges of redshift for the $N=512$ and $N=768$ sets. However, or the $N=256$ set the new formula produces a systematically low value for $M_{c}$ even for the $v_{\mathrm{bc}}=0$ case. As mentioned in Paper I, this run suffers from low statistic, particularly in the large mass tail,. Furthermore, there was no convergence of the gas fraction, these are the main cause for this systematics. Therefore, based on the $N=512$ and $N=768$ sets, we conclude that although the new formula produces somewhat better fit, the final $M_{c}$ results did not changed by much. This is not surprising since the meaning of the two models is the same.

As shown in Figure 7, the new formula results in a plateau at the high mass tail, which indicates a possible degeneracy between the fitting parameters. This of course present a problem in evaluating the values and errors of $M_{c}$. However, as shown in Figure 8 the values of $M_{c}$ from the two fitting models are consistent. Therefore, in evaluating the errors of the fitting values of $M_{c}$ we choose to be conservative and select the larger values between the $1-\sigma$ errors from the two models. In addition we have used bootstrap method for the new model, in some cases of the $N=512$ set to test our evaluation of the errors, and found that they are consistent with choosing the maximum $1-\sigma$ from the two fit models. We show the complete best fit parameters for the 512 set in table 2, they are similar for the other sets. Note that for high redshift and large $v_{\mathrm{bc}}$ values the best fit parameters are poorly constrain. Specifically the parameters $\beta$ and $\gamma$ from equation (2) are sometimes so poorly constrains (i.e., more then an order of magnitude) that we omit the errors from the table, thus the symbol "--" in table 2 means error larger then an order of magnitude, for those cases $\chi^{2}$ was close to zero.

Note that in our calculation of the fit we used $f_{\mathrm{b}, 0}$ which is the gas fraction in the high mass tail. This value is lower than the mean cosmic baryonic fraction $\bar{f}_{\mathrm{b}}$, since the baryons are lagging behind the dark matter even at high redshifts (see Naoz \& Barkana 2007; Barkana \& Loeb 2011). As shown in Naoz et al. 2011, fig. 4), this causes a higher $f_{\mathrm{b}, 0}$ for the initial conditions that assumes $\delta_{\mathrm{b}}=\delta_{\mathrm{dm}}$ than the $f_{\mathrm{b}, 0}$ resulted from the smother baryonic initial conditions,

\footnotetext{
${ }^{9}$ We could not use the bootstrap method to all of the runs since
} 


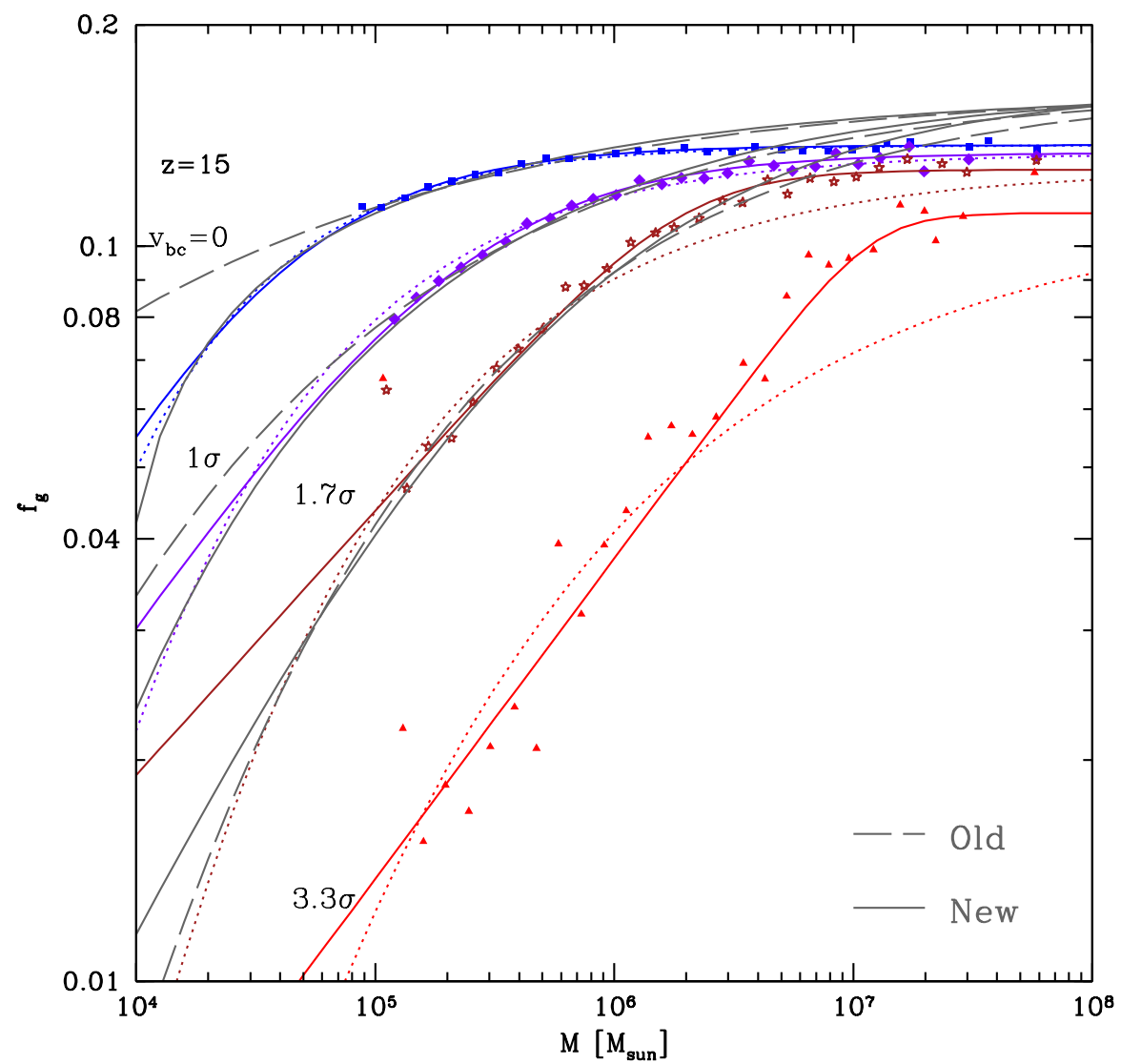

FIG. 9.- Comparison between the new fit model and the old one while using $f_{\mathrm{b}, 0}$ and $\bar{f}_{\mathrm{b}}$. We consider the $N=512$ run for $z=15$ representative example. We compare between using $\bar{f}_{\mathrm{b}}$ value, solid (dotted) grey lines for the new (old) model. We also show the fit while using the $f_{\mathrm{b}, 0}$ value, solid (dotted) color lines for the new (old) model. We consider the various values of the stream velocity $v_{\mathrm{bc}}=0$, $v_{\mathrm{bc}}=1 \sigma_{\mathrm{vbc}}, v_{\mathrm{bc}}=1.7 \sigma_{\mathrm{vbc}}$, and $v_{\mathrm{bc}}=3.4 \sigma_{\mathrm{vbc}}$ (blue squares, purple diamonds, brown stars and red triangles respectively).

as explored in their other two initial conditions model:10. In addition to the physical reason, a numerical reason may arise, since some baryons are in fact bound to the halo and we miss them due to our halo finder algorithm (\$2.3). As can be seen in Figure 9 assuming $f_{\mathrm{b}, 0} \rightarrow \bar{f}_{\mathrm{b}}$, grey lines, by either of the models results in a worse fit than using the calculated $f_{\mathrm{b}, 0}$. 
TABLE 2

The best-fit parameters from equation (2) For the $N=512$ Set.

\begin{tabular}{|c|c|c|c|}
\hline Redshift & $M_{c}\left[\mathrm{M}_{\odot}\right]$ & $\beta$ & $\gamma$ \\
\hline \multicolumn{4}{|l|}{$v_{\mathrm{bc}}=0$} \\
\hline 31 & $2.8_{-1.1}^{+2.2} \times 10^{4}$ & $1.45 \pm 0.71$ & $3.4 \pm 0.8$ \\
\hline 29 & $2.3_{-1 .}^{+1.1} \times 10^{4}$ & $1.09 \pm 0.1$ & $2.33 \pm 0.5$ \\
\hline 27 & $2_{-0.3}^{+1.3} \times 10^{4}$ & $1.37 \pm 0.1$ & $3.37 \pm 0.5$ \\
\hline 25 & $2_{-0.5}^{+0.6} \times 10^{4}$ & $1.14 \pm 0.06$ & $2.68 \pm 0.33$ \\
\hline 24 & $1.9_{-0.4}^{+0.0} \times 10^{4}$ & $1.15 \pm 0.06$ & $2.76 \pm 0.31$ \\
\hline 23 & $1.8_{-0.4}^{+0.6} \times 10^{4}$ & $1.04 \pm 0.04$ & $2.16 \pm 0.25$ \\
\hline 22 & $1.8_{-0.1}^{+0.7} \times 10^{4}$ & $1.17 \pm 0.05$ & $2.87 \pm 0.26$ \\
\hline 21 & $1.7_{-0.1}^{+0.6} \times 10^{4}$ & $1.15 \pm 0.04$ & $2.71 \pm 0.23$ \\
\hline 20 & $1.7_{-0.2}^{+0.4} \times 10^{4}$ & $1.05 \pm 0.03$ & $2.35 \pm 0.2$ \\
\hline 19 & $1.7_{-0.2}^{+0.2} \times 10^{4}$ & $0.96 \pm 0.03$ & $1.91 \pm 0.18$ \\
\hline 18 & $1.7_{-0.3}^{+0.2} \times 10^{4}$ & $0.91 \pm 0.02$ & $1.66 \pm 0.15$ \\
\hline 17 & $1.7_{-0.7}^{+0.4} \times 10^{4}$ & $0.87 \pm 0.02$ & $1.43 \pm 0.15$ \\
\hline 16 & $1.7_{-0.2}^{+0.2} \times 10^{4}$ & $0.88 \pm 0.02$ & $1.36 \pm 0.14$ \\
\hline 15 & $1.7_{-0.2}^{+0.2} \times 10^{4}$ & $0.91 \pm 0.02$ & $1.47 \pm 0.14$ \\
\hline \multicolumn{4}{|c|}{$v_{\mathrm{bc}}=1 \sigma_{\mathrm{vbc}}$} \\
\hline 29 & $1.2_{-0.2}^{+0.6} \times 10^{5}$ & $7.35 \pm 11.88$ & $15.78 \pm 25.79$ \\
\hline 27 & $1.3_{-0.3}^{+0.5} \times 10^{5}$ & $6.13 \pm 5.86$ & $14.14 \pm 13.78$ \\
\hline 25 & $1.3_{-0.3}^{+0.4} \times 10^{5}$ & $1.75 \pm 0.33$ & $3.89 \pm 0.91$ \\
\hline 24 & $1.3_{-0.4}^{+0.4} \times 10^{5}$ & $1.64 \pm 0.25$ & $3.55 \pm 0.69$ \\
\hline 23 & $1.2_{-0.2}^{+0.6} \times 10^{5}$ & 1.440 .19 & $3.2 \pm 0.57$ \\
\hline 22 & $1.2_{-0.3}^{+0.3} \times 10^{5}$ & $1.38 \pm 0.15$ & $3.1 \pm 0.47$ \\
\hline 21 & $1.1_{-0.3}^{+0.6} \times 10^{5}$ & $1.21 \pm 0.11$ & $2.7 \pm 0.38$ \\
\hline 20 & $1.1_{-0.3}^{+0.2} \times 10^{5}$ & $1.12 \pm 0.09$ & $2.52 \pm 0.32$ \\
\hline 19 & $9.9_{-2.6}^{+2.2} \times 10^{4}$ & $1.01 \pm 0.07$ & $2.19 \pm 0.28$ \\
\hline 18 & $9.5_{-2}^{+2.8} \times 10^{4}$ & $0.97 \pm 0.06$ & $2.17 \pm 0.26$ \\
\hline 17 & $8.1_{-1.8}^{+1.1} \times 10^{4}$ & $1.05 \pm 0.06$ & $2.4 \pm 0.26$ \\
\hline 16 & $7.2_{-1.4}^{+1.1} \times 10^{4}$ & 1.010 .05 & $2.22 \pm 0.23$ \\
\hline 15 & $6.8_{-1.8}^{+1.1} \times 10^{4}$ & 0.970 .05 & $2.15 \pm 0.22$ \\
\hline \multicolumn{4}{|c|}{$v_{\mathrm{bc}}=1.7 \sigma_{\mathrm{vbc}}$} \\
\hline 25 & $4_{-1.1}^{+1.3} \times 10^{5}$ & $4.45 \pm 6.74$ & $8.7 \pm 13.68$ \\
\hline 24 & $4.3_{-1.2}^{+1.3} \times 10^{5}$ & $3.94 \pm 5.16$ & $8.19 \pm 11.15$ \\
\hline 23 & $4_{-0.9}^{+1.3 .2} \times 10^{5}$ & $3.58 \pm 3.14$ & $7.36 \pm 6.8$ \\
\hline 22 & $4.7_{-1}^{+1.3} \times 10^{5}$ & $4.96 \pm 5.98$ & $11.03 \pm 13.63$ \\
\hline 21 & $4.6_{-0.7}^{+1.3} \times 10^{5}$ & 2.912 & $6.89 \pm 5.05$ \\
\hline 20 & $4.6_{-0.9}^{+1.2} \times 10^{5}$ & $3.45 \pm 2.35$ & $8.5 \pm 6.06$ \\
\hline 19 & $4.5_{-0.7}^{+1.2} \times 10^{5}$ & $2.64 \pm 1.23$ & $6.7 \pm 3.36$ \\
\hline 18 & $4.3_{-1}^{+1.2} \times 10^{5}$ & $2.06 \pm 0.69$ & $5.26 \pm 1.97$ \\
\hline 17 & $3.6_{-0.7}^{+1.2} \times 10^{5}$ & $2.07 \pm 0.7$ & $5.69 \pm 2.16$ \\
\hline 16 & $3.2_{-0.8}^{+1.1} \times 10^{5}$ & $1.75 \pm 0.41$ & $4.7 \pm 1.31$ \\
\hline 15 & $2.9_{-0.6}^{+0.3} \times 10^{5}$ & $1.77 \pm 0.38$ & $4.9 \pm 1.25$ \\
\hline \multicolumn{4}{|c|}{$v_{\mathrm{bc}}=3.4 \sigma_{\mathrm{vbc}}$} \\
\hline 20 & $2.7_{-1.7}^{+2.3} \times 10^{6}$ & $102--$ & $222--$ \\
\hline 19 & $3_{-1.7}^{+1.2} \times 10^{6}$ & $88--$ & $187--$ \\
\hline 18 & $3_{-1.1}^{+1.1} \times 10^{6}$ & $4.82 \pm 13.1$ & $10.3--$ \\
\hline 17 & $3.4_{-1.6}^{+0.7} \times 10^{6}$ & $3.56 \pm 7.9$ & $8.16 \pm 18.4$ \\
\hline 16 & $3.2_{-1.4}^{+0.0} \times 10^{6}$ & $2.1 \pm 1.23$ & $4.8 \pm 2.9$ \\
\hline 15 & $2.6_{-0.5}^{+0.9} \times 10^{6}$ & $2.33 \pm 1.35$ & $5.3 \pm 3.2$ \\
\hline
\end{tabular}

